\title{
Reducing Interface Recombination through Mixed Nanocrystal Interlayers in PbS Quantum Dot Solar Cells
}

Santanu Pradhan ${ }^{1}$, Alexandros Stavrinadis ${ }^{1}$, Shuchi Gupta ${ }^{1}$, and Gerasimos Konstantatos ${ }^{1,2 *}$

${ }^{1}$ ICFO-Institut de Ciencies Fotoniques, The Barcelona Institute of Science and Technology, 08860 Castelldefels (Barcelona), Spain

${ }^{2}$ ICREA-Institució Catalana de Recerca i Estudis Avançats, Passeig Lluís Companys 23, 08010 Barcelona, Spain

* gerasimos.konstantatos@,icfo.es

Abstract: The performance of $\mathrm{ZnO} / \mathrm{PbS}$ colloidal quantum dot (CQD) based heterojunction solar cells is hindered by charge carrier recombination at the heterojunction interface. Reducing interfacial recombination can improve charge collection and the photocurrent of the device. Here we report the use of a mixed nanocrystal (MNC) buffer layer comprising zinc oxide nanocrystals and lead sulfide quantum dots at the respective heterojunction interface. Remote trap passivation of the PbS CQDs taking place within this MNC layer reduces interfacial recombination, electron back transfer and improves charge collection efficiency. Upon the addition of the MNC layer, the overall power conversion efficiency increases from $9.11 \%$ to $10.16 \%$ and Short-circuit current density $\left(J_{S C}\right)$ increases from $23.54 \mathrm{~mA} / \mathrm{cm}^{2}$ to $25.23 \mathrm{~mA} / \mathrm{cm}^{2}$. Optoelectronic characterization of the solar cells confirms that the effects underlying device improvement are reduced trap density and improved charge collection efficiency due to the presence of the MNC buffer layer.

Keywords: colloidal quantum dots, $\mathrm{PbS}$, interface recombination, photovoltaics, mixed nanocrystals 


\section{Introduction:}

Solution processed colloidal quantum dot (CQD) based photovoltaics (PV) have attracted significant research interest due to the advantages of low-cost solution processibility and optical properties tunability of $\mathrm{CQDs}^{1-3}$. In particular, rapid progress made in $\mathrm{ZnO} / \mathrm{PbS}$ CQD based solar cells has been facilitated by optimizing device architectures ${ }^{4}$, understanding the device physics ${ }^{5}$ and improving the electronic properties and surface passivation of QD films and devices ${ }^{6,7}$. As a result, the power conversion efficiency (PCE) of CQD solar cells improved from $1 \%$ to over $11 \%$ in the last decade ${ }^{8,9}$.

In spite of this tremendous progress, further improvements to suppress the various loss mechanisms that hinder the performance of these devices is still in search. Such a loss mechanism is the undesired interface recombination of photo-generated charge carriers. While non-radiative recombination in the PbS QD solids plays a major role in the open circuit voltage $\left(V_{O C}\right)$ deficit $^{10-12}$, interfacial recombination at the $\mathrm{ZnO} / \mathrm{PbS}$ heterojunction influences the charge collection and hence the $\mathrm{PCE}^{13}$. High efficiency $\mathrm{PbS}$ quantum dot solar cells are based on a depleted heterojunction formed between the transparent n-type $\mathrm{ZnO}$ layer and the photoabsorbing p-type (with respect to $\mathrm{ZnO}$ doping) $\mathrm{PbS}$ QD layer. Electron collection at the transparent electrode depends on the efficient electron transfer from $\mathrm{PbS}$ to $\mathrm{ZnO}$ and reduction of electron back transfer from $\mathrm{ZnO}$ to $\mathrm{PbS}$. The latter is even more relevant when the solar cell is operated under realistic conditions in the maximum power point. Efficient electron transfer from $\mathrm{PbS}$ to the $\mathrm{ZnO}$ depends on the rate of charge transfer and the rate of interface recombination ${ }^{14}$. It is thus important to improve the charge transfer rate and decrease interfacial recombination for improved charge collection, particularly under maximum power point (MPP) operation. The reduction of the interfacial charge carrier recombination can be achieved via the reduction of interfacial charge carrier density and recombination velocity ${ }^{15}$. These parameters are influenced by the presence of defects in $\mathrm{ZnO}$ located in the vicinity of the 
junction. Suppressing these defects can reduce the interfacial recombination and hence can enhance charge collection at the electrode.

In the past, several techniques have been adopted to reduce the charge carrier recombination at the interface. For $\mathrm{TiO}_{2} / \mathrm{PbS}$ solar cells, a thin $\mathrm{ZnO}$ layer over $\mathrm{TiO}_{2}$ has been reported as an efficient buffer layer for reducing interface recombination ${ }^{16}$. Yuan et. al. showed that n-type PCBM ([6,6]-phenyl-61-butyric acid) can be an efficient buffer layer to replace the $\mathrm{ZnO}$ layer as it energetically fits in between the $\mathrm{n}$-type and p-type layer of the heterojunction ${ }^{17}$. Doped $\mathrm{ZnO}$ has also been presented as an efficient buffer layer ${ }^{18}$ which changes favourably the n-type doping concentration at the electron accepting side of the device ${ }^{19}$. Recently, Zhao et. al. have demonstrated a CdSe buffer layer in PbS QD solar cell following the well-known CIGS solar cell buffer layer approach where band bending energetically helping in the smooth conduction of electron from $\mathrm{PbS}$ to $\mathrm{ZnO}^{20}$. Overall, an ideal interfacial buffer layer must be trap-free, nearly intrinsic as well as energetically favourable to fit in between $\mathrm{ZnO}$ and $\mathrm{PbS}$, and such layer had thus far remained elusive. Instead of relying on the employment of other materials, in this work we report the use of a mixed nanocrystalline (MNC) layer comprising $\mathrm{ZnO}$ nanocrystals (NCs) and $\mathrm{PbS}$ CQDs i.e. the individual components of the heterojunction as a buffer layer that fulfils the aforementioned desired characteristics. Such a mixed nanocrystalline composite has been reported in the past to act as a nearly trap free material when used as a bulk heterojunction quantum dot layer ${ }^{21,22}$, achieving very high Voc and approaching the band-to-band recombination regime.

The MNC layer is prepared using an optimized mixing weight ratio of $\mathrm{PbS}$ and $\mathrm{ZnO}$ of 2:1 (details in experimental section of supporting information). The buffer layer was treated with MPA (3-mercaptopropionic acid, 5\% in methanol) solution similar to the previous report $^{21}$. To investigate the effect of the MNC layer as a buffer layer, we have compared the MNC-buffer-layer device with the reference solar cell where the active layer PbS QDs are 
treated with 1-ethyl-3-methylimidazolium iodide (EMII) and the top electron blocking layer is treated with 1, 2 ethane dithiol (EDT) ${ }^{23}$.

\section{Results \& Discussions:}

Figure 1(a) is a schematic of the respective energy band alignment in short-circuit condition with the presence of MNC buffer layer. The buffer layer helps in electron transport with a favourable energy landscape along with a reduced trap state density and electron back transfer from $\mathrm{ZnO}$ to the $\mathrm{PbS}$ main absorber. The optimized thickness of the buffer layer is found to be $10 \mathrm{~nm}$ (1 layer). Supporting Figure S1 shows the effect of thickness variation of MNC buffer layer on the device performance compared to the reference device. Increasing the thickness of the MNC layer from 10 to $40 \mathrm{~nm}$ decreases gradually the PCE of the devices. The major reason for this decrement is associated with the decrease of both short circuit current density ( $\mathrm{JSC}_{\mathrm{SC}}$ and fill factor (FF). The FF is highly influenced by the increase of series resistance $\left(\mathrm{R}_{\mathrm{s}}\right)$ upon increasing the thickness of the MNC layer. Interestingly, the open circuit voltage (VOC) of the devices with the MNC layer improved slightly. This indicates that the MNC buffer layer facilitates the decrease interface charge recombination. The thickness of the EMII treated layer for both the devices was approximately 180-190 nm and the EDT layer thickness was close to 30-35 nm whereas the thickness of the $\mathrm{ZnO}$ layer was approximately $50 \mathrm{~nm}$. The FIB SEM (scanning electron microscopy) figures for devices with and without the MNC buffer layer are shown in Supporting Figure S2.

The efficiency of the solar cells are estimated from the current density-voltage (J-V) plot as depicted in Figure 1(b). Table 1 summarises the photovoltaic figures of merits for the reference and the MNC buffer layer containing devices. The incorporation of the MNC layer improves $\mathrm{J}_{\mathrm{SC}}$ from $23.54 \mathrm{~mA} / \mathrm{cm}^{2}$ to $25.23 \mathrm{~mA} / \mathrm{cm}^{2}$ whereas the $\mathrm{V}_{\mathrm{OC}}(0.6 \mathrm{~V}$ to $0.61 \mathrm{~V})$ and $\mathrm{FF}$ (0.65 to 0.66 ) also improved slightly. Overall, the PCE improves from $8.80 \pm 0.24 \%$ to 
$9.87 \pm 0.17 \%$ (from $9.11 \%$ to $10.16 \%$ for the champion devices). It is noteworthy that with the incorporation of the MNC buffer layer, series resistance $\left(\mathrm{R}_{\mathrm{s}}\right)$ increased from $96 \Omega$ to $103 \Omega$ whereas the shunt resistance $\left(\mathrm{R}_{\mathrm{sh}}\right)$ improved from $22.40 \mathrm{k} \Omega$ to $26.18 \mathrm{k} \Omega$ which indicates that the MNC buffer layer decreases current leakage at the heterojunction interface. The decrease of leakage current and the improvement of $\mathrm{V}_{\text {OC }}$ reflects also on the dark current- voltage analysis as shown in Figure 1(c). VOC of the solar cell can be expressed as, $V_{O C} \approx \frac{\eta k T}{q} \ln \left(\frac{J_{p h}}{J_{0}}\right)$. Where $\boldsymbol{\eta}$ is the diode ideality factor, $\mathrm{J}_{0}$ is the reverse saturation current density and $\mathrm{J}_{\mathrm{ph}}$ is the photo-generated current density. Decrease of $\mathrm{J}_{0}$ is observed with $\mathrm{MNC}$ buffer layer incorporation which indicates the reduction leakage current and slight increase in Voc. The decrease in calculated $\boldsymbol{\eta}$ (1.64 to 1.55 with MNC layer incorporation) from the dark current also indicates the reduction of interfacial recombination in the device. We have further performed Mott-Schottky analysis of capacitance-voltage $(\mathrm{C}-\mathrm{V})$ of our devices in an attempt to understand how the MNC buffer layer impacts the built-in potential ( $\left.\mathrm{V}_{\mathrm{bi}}\right)$ and other characteristic of the heterojunction. Supporting Figure $S 3$ shows the $1 / C^{2}-V$ measurements (and respective linear fits) of the devices. From there we extrapolate that the MNC buffer layer improves $\mathrm{V}_{\mathrm{bi}}$ from $0.63 \mathrm{~V}$ to $0.68 \mathrm{~V}$. The improved $\mathrm{V}_{\mathrm{bi}}$ allows for more efficient charge transfer and charge collection.

$\mathrm{J}_{\mathrm{SC}}$ improves with the addition of the MNC layer because the latter improves charge collection. To verify this, we investigated the external quantum efficiency (EQE) of the devices with and without an electrical bias. In accordance to previous reports, the external bias is set to the value corresponding to the maximum power point (MPP) of the device ${ }^{24}$. The EQE spectra without an applied bias are shown in Figure 2(a). These confirm that MNC incorporation improves the devices' JSC as the integrated JSC from EQE spectra for MNC buffer layer is 21.7 $\mathrm{mA} / \mathrm{cm}^{2}$ compared to $19.8 \mathrm{~mA} / \mathrm{cm}^{2}$ of reference device. We attribute the apparent change in the shape of EQE spectrum between these two devices to the interference peak shift with the 
optical thickness change due to the incorporation of MNC layer. The EQE spectra for the MPP points of the devices are shown in Figure 2(b). The integrated $\mathrm{J}_{\mathrm{SC}}$ from EQE for reference showed much degraded value of $13.7 \mathrm{~mA} / \mathrm{cm}^{2}$ compared to $19.4 \mathrm{~mA} / \mathrm{cm}^{2}$ for the MNC buffer layer incorporated device. The difference between the EQE of reference and MNC layer incorporated devices with and without MPP-bias are shown in Figure 2(c), where we observe that this difference is increased when the MNC buffer layer is present. This confirms that the MNC buffer layer reduces interfacial recombination and enhances charge carrier collection efficiency even under low built-in electric field of the junction, as is the case under the MPP condition. Moreover, the screening of $\mathrm{MNC}$ layer over the $\mathrm{ZnO}$ has a significant effect on the hysteresis of the I-V curve. Supporting Figure S4 (a) \& (b) show the hysteresis behaviour of the I-V curves for reference and MNC interface devices. It is observed that the MNC buffer layer dramatically decreases hysteresis. This can be attributed to the fact that the charge accumulation and transfer at the heterojunction with different voltage bias is influenced by the presence of interfacial traps ${ }^{25}$. The presence of such traps result in accumulation of photogenerated carrier at the heterojunction interface which subsequently cause significant hysteresis during the J-V scan. This charge accumulation and associated hysteresis is greatly suppressed by inserting the MNC layer.

We have also investigated the effect of MNC buffer layer on the stability of the cells. Figure 3 illustrates the evolution of different PV parameters for reference device and the MNC buffer layer inserted device (champion devices in both cases) up to 40 days stored and measured in ambient conditions. For both cases, $\mathrm{V}_{\mathrm{OC}}$ and $\mathrm{J}_{\mathrm{SC}}$ increased with time and then saturated similar to the earlier reported results ${ }^{4}$. Interestingly, the FF of the reference device decreased with time contrary to the MNC buffer layer inserted device which demonstrates a remarkably stable FF. Overall, the efficiency of the reference device slightly decreased after 40 days whereas the buffer layer improves the stability as shown in Figure 3(d). The major 
reason for this improvement attributed to the stable value of FF over the course of the period. The insertion of the buffer layer at the heterojunction reduced recombination induced leakage and hence improve FF. This also supports previously explained reduction of hysteresis loss and field induced interfacial recombination.

We sought to further understand the effect of MNC buffer layer on charge collection and recombination. Intensity $(\Phi)$ dependent photocurrent $\left(J_{p h}\right)$ variation shows that both reference and MNC devices have linear power dependency $\left(J_{p h} \propto \Phi^{p}, p=1\right)$ (Figure 4(a)). The diode ideality factor $(\boldsymbol{\eta})$ of the devices is estimated from the intensity dependent $V_{O C}$ relation, $V_{O C} \approx \frac{\eta k T}{q} \ln (\Phi)$, where $\mathrm{kT}$ is the thermal energy and $\mathrm{q}$ is the elementary charge (Figure 4(b)). The estimated $\boldsymbol{\eta}$ for the reference device is 1.51 which is consistent with previously reported PbS QD based depleted heterojunction solar cells. $\boldsymbol{\eta}$ close to 1 indicates the transition into band to band recombination regime whereas $\boldsymbol{\eta}$ in between 1 and 2 indicates trap induced recombination ${ }^{26}$. Inserting the MNC layer reduced the value of $\boldsymbol{\eta}$ to 1.38 . This reduction in $\boldsymbol{\eta}$ follows a similar trend in the aforementioned dark current method which indicates the reduction of interfacial recombination with the buffer layer.

The charge carrier recombination dynamics of the solar cells were further investigated with combined transient photo-voltage (TPV) and photocurrent (TPC) measurements. Recombination lifetime $\left(\boldsymbol{\tau}_{\mathrm{r}}\right)$ was calculated from an exponential fit on the photo-voltage transient curve. The TPV and TPC plot for both devices at a VOC bias of $0.41 \mathrm{~V}$ is shown in Supporting Figure S5. The variation of $\boldsymbol{\tau}_{\mathrm{r}}$ with Voc is plotted in Figure 4(c) and it shows that the recombination is slowed by the addition of the MNC buffer layer. Moreover, the photogenerated charge carriers are estimated by combining TPV and TPC data ${ }^{26-29}$ and this information is used for calculating the recombination rate by dividing the charge density by 
recombination lifetime as depicted in Figure 4(d). Therein it is evident that the MNC interface device benefits from a lower recombination rate in agreement with our original hypothesis.

\section{Conclusions:}

We have shown that the insertion of a thin $\mathrm{ZnO} / \mathrm{PbS}$ QD MNC layer acts as an efficient interfacial buffer layer in $\mathrm{ZnO} / \mathrm{PbS}$ heterojunction solar cells. Various device characterization measurements confirm the effectiveness of the MNC buffer layer for supressing interface recombination, improving charge collection at the respective side of the heterojunction, and ultimately for improving all the photovoltaic figures of merit of the solar cells. The improved photovoltaic performance and stability of the devices, combined with the simple solution processibility of the MNC buffer layer, renders this concept an attractive option to be considered for improving various other types of nanostructured heterojunction solar cells.

\section{Acknowledgements}

We acknowledge financial support from the European Research Council (ERC) under the European Union's Horizon 2020 research and innovation programme (grant agreement No 725165), the Spanish Ministry of Economy and Competitiveness (MINECO) and the "Fondo Europeo de Desarrollo Regional" (FEDER) through grant MAT2014-56210-R. This work was also supported by AGAUR under the SGR grant (2014SGR1548) and by European Union H2020 Programme under grant agreement ${ }^{\circ} 696656$ Graphene Flagship. We also acknowledge financial support from Fundacio Privada Cellex, the CERCA Programme and the Spanish Ministry of Economy and Competitiveness, through the "Severo Ochoa" Programme for Centres of Excellence in R\&D (SEV-2015-0522). 


\section{Supporting information:}

Experimental details; MNC layer thickness optimization; FIB SEM device micrograph; C-V analysis; Hysteresis of the J-V plots; TPV \& TPC plots.

\section{References:}

(1) Carey, G. H.; Abdelhady, A. L.; Ning, Z.; Thon, S. M.; Bakr, O. M.; Sargent, E. H. Colloidal Quantum Dot Solar Cells. Chem. Rev. 2015, 115, 12732-12763.

(2) Sargent, E. H. Colloidal Quantum Dot Solar Cells. Nat. Photonics, 2012, 6, 133-135.

(3) Nozik, A. J.; Beard, M. C.; Luther, J. M.; Law, M.; Ellingson, R. J.; Johnson, J. C. Semiconductor Quantum Dots and Quantum Dot Arrays and Applications of Multiple Exciton Generation to Third-Generation Photovoltaic Solar Cells. Chem. Rev. 2010, 110, 6873-6890.

(4) Chuang, C.-H. M.; Brown, P. R.; Bulović, V.; Bawendi, M. G. Improved performance and stability in quantum dot solar cells through band alignment engineering. Nat. Mater.

2014, 13, 796-801.

(5) Kagan, C. R.; Lifshitz, E.; Sargent, E. H.; Talapin, D. V. Building Devices from Colloidal Quantum Dots. Science, 2016, 353, 885.

(6) Jeong, K.S; Tang, J.; Liu, H.; Kim, J.; Schaefer, A. W; Kemp, K.; Levina, L.; Wang, X.; Hoogland, S.; Debnath, R.; Brzozowski, L.; Sargent, E. H.; Asbury, J. B. Enhanced MobilityLifetime Products in PbS Colloidal Quantum Dot Photovoltaics. ACS Nano, 2012, 6, 89-99.

(7) Brown, P. R., Kim, D.; Lunt, R. R.; Zhao, N.; Bawendi, M. G.; Grossman, J. C.; Bulovic, V. Energy Level Modification in Lead Sulfide Quantum Dot Thin Films through Ligand Exchange. ACS Nano, 2014, 8, 5863-5872.

(8) McDonald, S. A.; Konstantatos, G; Zhang, S.; Cyr, P. W.; Klem, E.J.D.; Levina, L.; Sargent, E.H. Solution-Processed PbS Quantum Dot Infrared Photodetectors and Photovoltaics. Nat. Mater. 2005, 4, 138-142.

(9) Liu, M.; Voznyy, O.; Sabatini, R.; Garcia de Arquer, F. P.; Munir, R.; Balawi, A. H.; Lan, X.; Fan, F.; Walters, G.; Kirmani, A. R.; Hoogland, S.; Laquai, F.; Amassian, A; Sargent, E. H. Hybrid Organic-Inorganic Inks Flatten the Energy Landscape in Colloidal Quantum Dot Solids. Nat. Mater. 2017, 16, 258-263.

(10) Chuang, C.-H. M.; Maurano, A.; Brandt, R. E.; Hwang, G. W.; Jean, J.; Buonassisi, T; Bulović, V.; Bawendi, M. G. Open-Circuit Voltage Deficit, Radiative Sub-Bandgap States, and Prospects in Quantum Dot Solar Cells. Nano Lett., 2015, 15, 3286-3294. 
(11) Stavrinadis, A.; Pradhan, S.; Papagiorgis, P.; Itskos, G.; Konstantatos, G. Suppressing Deep Traps in PbS Colloidal Quantum Dots via Facile Iodide Substitutional Doping for Solar Cells with Efficiency $>10 \%$. ACS Energy Lett. 2017, 2, 739-744.

(12) Pradhan, S.; Stavrinadis, A.; Gupta, S.; Bi, Y.; Di Stasio, F.; Konstantatos, G. TrapState Suppression and Improved Charge Transport in PbS Quantum Dot Solar Cells with Synergistic Mixed-Ligand Treatments. Small, 2017 (DOI: 10.1002/smll.201700598).

(13) Byers, J. C.; Ballantyne, S.; Rodionov, K.; Mann, A.; Semenikhin, O. A. Mechanism of Recombination Losses in Bulk Heterojunction P3HT:PCBM Solar Cells Studied Using Intensity Modulated Photocurrent Spectroscopy. ACS Appl. Mater. Interfaces 2011, 3, 392401.

(14) Tan, F.; Wang, Z.; Qu, S.; Cao, D.; Liu, K.; Jiang, Q.; Yang, Y.; Pang, S.; Zhang, W.; Lei, Y.; Wangb, Z. A CdSe Thin Film: A Versatile Buffer Layer for Improving the Performance of $\mathrm{TiO}_{2}$ Nanorod Array: PbS Quantum Dot Solar Cells. Nanoscale, 2016, 8, 10198-10204.

(15) Kemp, K. W.; Labelle, A. J.; Thon, S. M.; Ip, A. H.; Karmer, I. J.; Hoogland, S.; Sargent, E. H. Interface Recombination in Depleted Heterojunction Photovoltaics based on Colloidal Quantum Dots. Adv. Energy. Mater. 2013, 3, 917-922.

(16) Chang, J.; Kuga, Y.; Mora-Seró, I.; Toyoda, T.; Ogomi, Y.; Hayase, S.; Bisquert, J.; Shen, Q. High Reduction of Interfacial Charge Recombination in Colloidal Quantum Dot Solar Cells by Metal Oxide Surface Passivation. Nanoscale, 2015, 7, 5446-5456.

(17) Yuan, M.; Voznyy, O.; Zhitomirsky, D.; Kanjanaboos, P.; Sargent, E. H. Synergistic Doping of Fullerene Electron Transport Layer and Colloidal Quantum Dot Solids Enhances Solar Cell Performance. Adv. Mater. 2015, 27, 917-921.

(18) Zhang, X.; Johansson, E. M. J. Reduction of Charge Recombination in PbS Colloidal Quantum Dot Solar Cells at the Quantum Dot/ZnO Interface by Inserting a MgZnO Buffer Layer. J. Mater. Chem. A, 2017, 5, 303-310.

(19) Ehrler, B.; Musselman, K. P.; Böhm, M. L.; Morgenstern, F. S. F.; Vaynzof, Y.; Walker, B. J.; MacManus-Driscoll, J. L.; Greenham, N. C. Preventing Interfacial Recombination in Colloidal Quantum Dot Solar Cells by Doping the Metal Oxide. ACS Nano, 2013, 7, 4210 4220 .

(20) Zhao, T.; Goodwin, E. D.; Guo, J; Wang, H.; Diroll, B. T.; Murray, C. B; Kagan, C. R. Advanced Architecture for Colloidal PbS Quantum Dot Solar Cells Exploiting a CdSe Quantum Dot Buffer Layer. ACS Nano, 2016, 10, 9267-9273.

(21) Rath, A. K.; Garcia de Arquer, F. P.; Stavrinadis, A.; Lasanta, T.; Bernechea, M.; Diedenhofen, S. L.; Konstantatos, G. Remote Trap Passivation in Colloidal Quantum Dot Bulk Nano-heterojunctions and Its Effect in Solution-Processed Solar Cells. Adv. Mater. 2014, 26, 4741- 4747 . 
(22) Pradhan, S.; Stavrinadis, A.; Gupta, S.; Christodoulou, S.; Konstantatos, G. Breaking the Open-circuit Voltage Deficit Floor in PbS Quantum Dot Solar Cells Through Synergistic Ligand and Architecture Engineering, ACS Energy Lett. 2017, 2, 1444 - 1449.

(23) Cao, Y.; Stavrinadis, A.; Lasanta, T.; So, D.; Konstantatos, G. The Role of Surface Passivation for Efficient and Photostable PbS Quantum Dot Solar Cells. Nat. Energy, 2016, 1,16035 .

(24) Liu, M.; García de Arquer, F. P.; Li, Y.; Lan, X.; Kim, G.; Voznyy, O.; Jagadamma, L. K.; Abbas, A. S.; Hoogland, S.; Lu, Z.; Kim, J. Y.; Amassian, A.; Sargent, E. H. DoubleSided Junctions Enable High-Performance Colloidal-Quantum-Dot Photovoltaics. Adv. Mater. 2016, 28, 4142-4148.

(25) Kim, H.-S.; Jang, I.-H.; Ahn, N.; Choi, M.; Guerrero, A.; Bisquert, J.; Park, N.-G. Control of IV Hysteresis in $\mathrm{CH}_{3} \mathrm{NH}_{3} \mathrm{PbI}_{3}$ Perovskite Solar Cell. J. Phys. Chem. Lett. 2015, 6, 4633-4639.

(26) Zhao, N.; Osedach, T. P.; Chang, L.-Y.; Geyer, S. M.; Wanger, D.; Binda, M. T.; Arango, A. C.; Bawendi, M. G.; Bulovic, V. Colloidal PbS Quantum Dot Solar Cells with High Fill Factor. ACS Nano, 2010, 4, 3743-3752.

(27) Shuttle, C. G.; O’Regan, B.; Ballantyne, A. M.; Nelson, J; Bradley, D. D. C; de Mello, J; Durrant, J. R. Experimental Determination of the Rate Law for Charge Carrier Decay in a Polythiophene: Fullerene Solar Cell. Appl. Phys. Lett., 2008, 92, 093311.

(28) Chang, J.; Kuga, Y.; Mora-Seró, I.; Toyoda, T.; Ogomi, Y.; Hayase, S.; Bisquert, J.; Shen, Q. High Reduction of Interfacial Charge Recombination in Colloidal Quantum Dot Solar Cells by Metal Oxide Surface Passivation, Nanoscale, 2015, 7, 5446-5456.

(29) Zaban, A.; Greenshtein, M.; Bisquert, J. Determination of the Electron Lifetime in Nanocrystalline Dye Solar Cells by Open-Circuit Voltage Decay Measurements, Chem. Phys. Chem., 2003, 4, 859-864. 


\begin{tabular}{ccccccc}
\hline Device & $\boldsymbol{V}_{\text {OC }}(\boldsymbol{V})$ & $\begin{array}{c}\boldsymbol{J}_{\text {SC }} \\
\left(\mathbf{m A} / \mathbf{c m}^{2}\right)\end{array}$ & $\boldsymbol{F F}$ & $\begin{array}{c}\text { Efficiency } \\
(\%)\end{array}$ & $\boldsymbol{R}_{\boldsymbol{s}}(\Omega)$ & $\boldsymbol{R}_{\text {sh }}(\mathbf{k} \Omega)$ \\
\hline Reference & $\begin{array}{c}0.593 \pm .005 \\
(0.60)\end{array}$ & $\begin{array}{c}23.26 \pm 0.30 \\
(23.54)\end{array}$ & $\begin{array}{c}0.640 \pm 0.011 \\
(0.65)\end{array}$ & $\begin{array}{c}8.80 \pm 0.24 \\
(9.11)\end{array}$ & $\begin{array}{c}96 \pm 14 \\
(99)\end{array}$ & $\begin{array}{c}21.00 \pm 2.73 \\
(22.40)\end{array}$ \\
$\begin{array}{c}\text { With } \boldsymbol{M N C} \\
\text { interface }\end{array}$ & $\begin{array}{c}0.605 \pm .005 \\
(0.61)\end{array}$ & $\begin{array}{c}25.06 \pm 0.13 \\
(25.23)\end{array}$ & $\begin{array}{c}0.651 \pm .005 \\
(0.66)\end{array}$ & $\begin{array}{c}9.87 \pm 0.17 \\
(10.16)\end{array}$ & $\begin{array}{c}103 \pm 5 \\
(96)\end{array}$ & $\begin{array}{c}25.33 \pm 2.54 \\
(26.18)\end{array}$ \\
\hline
\end{tabular}

Table 1: Summary of photovoltaic figures of merit for Reference PbS QD based and MNC interfacial buffer layer incorporated devices (average over 6 devices for each case). The values in brackets indicate the champion device. 
Figures:
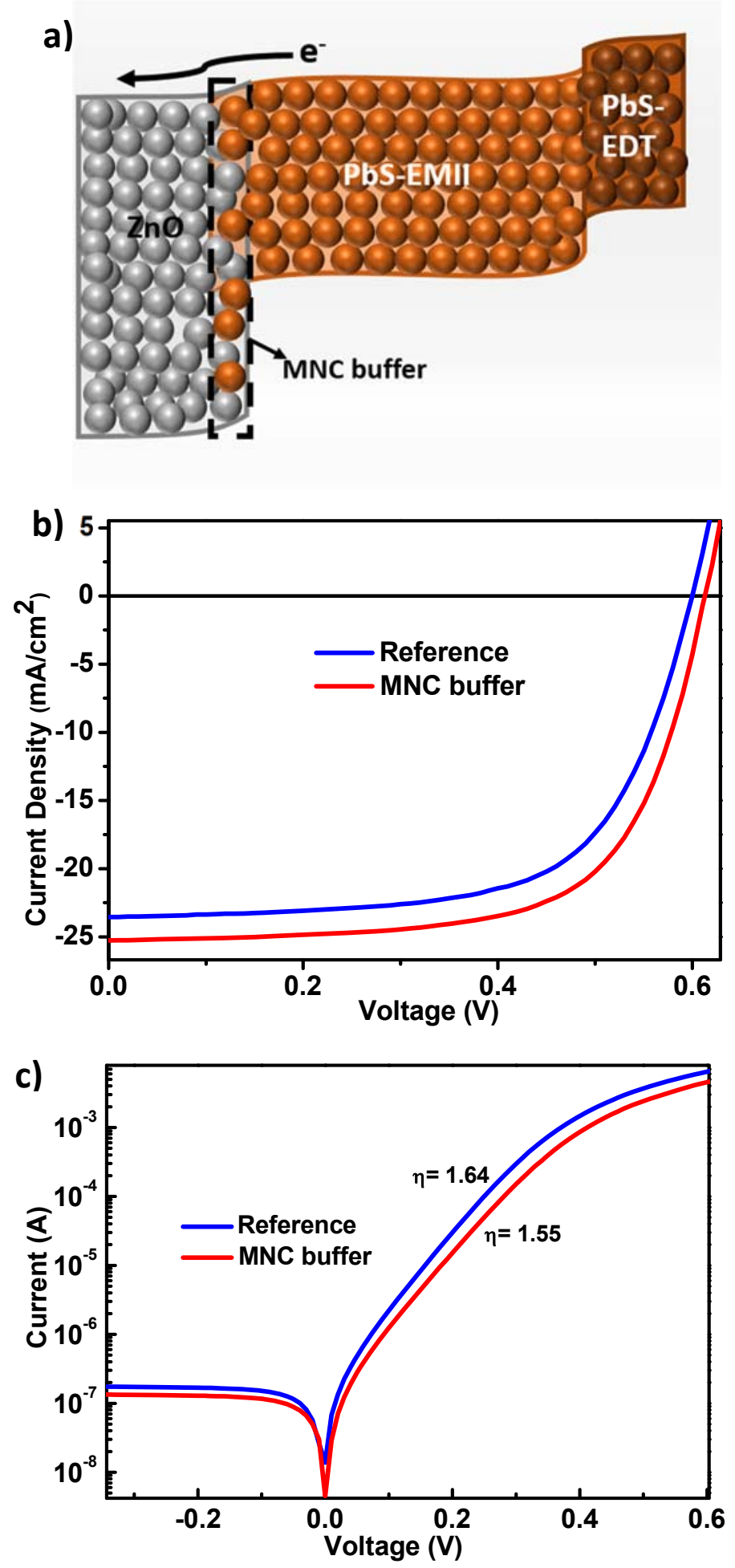

Figure 1: (a) Schematic energy band alignment of the device with inserting MNC layer in shortcircuit condition. The MNC layer facilitated electron transport and increases charge collection efficiency. (b) Photovoltaic performance of Reference and MNC buffer layer based device. (c) Dark current of Reference and MNC buffer layer inserted device. 

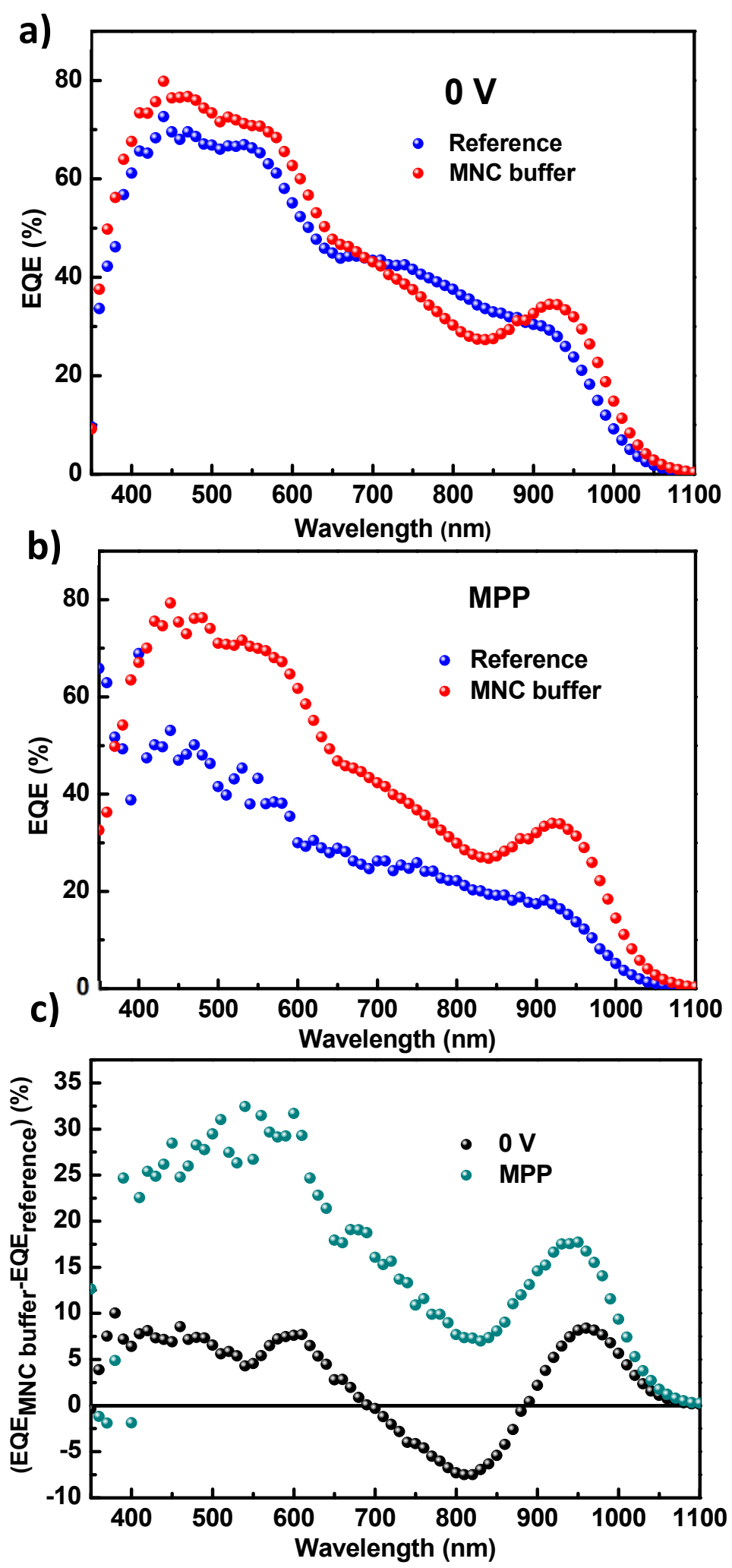

Figure 2: EQE spectra for both devices at (a) $0 \mathrm{~V}$ and (b) at MPP (maximum power point) bias. (c) Spectral EQE difference between reference and MNC buffer layer-device at $0 \mathrm{~V}$ and MPP. Voltage bias dependence of charge collection is less effective with MNC buffer layer incorporation. 
a)
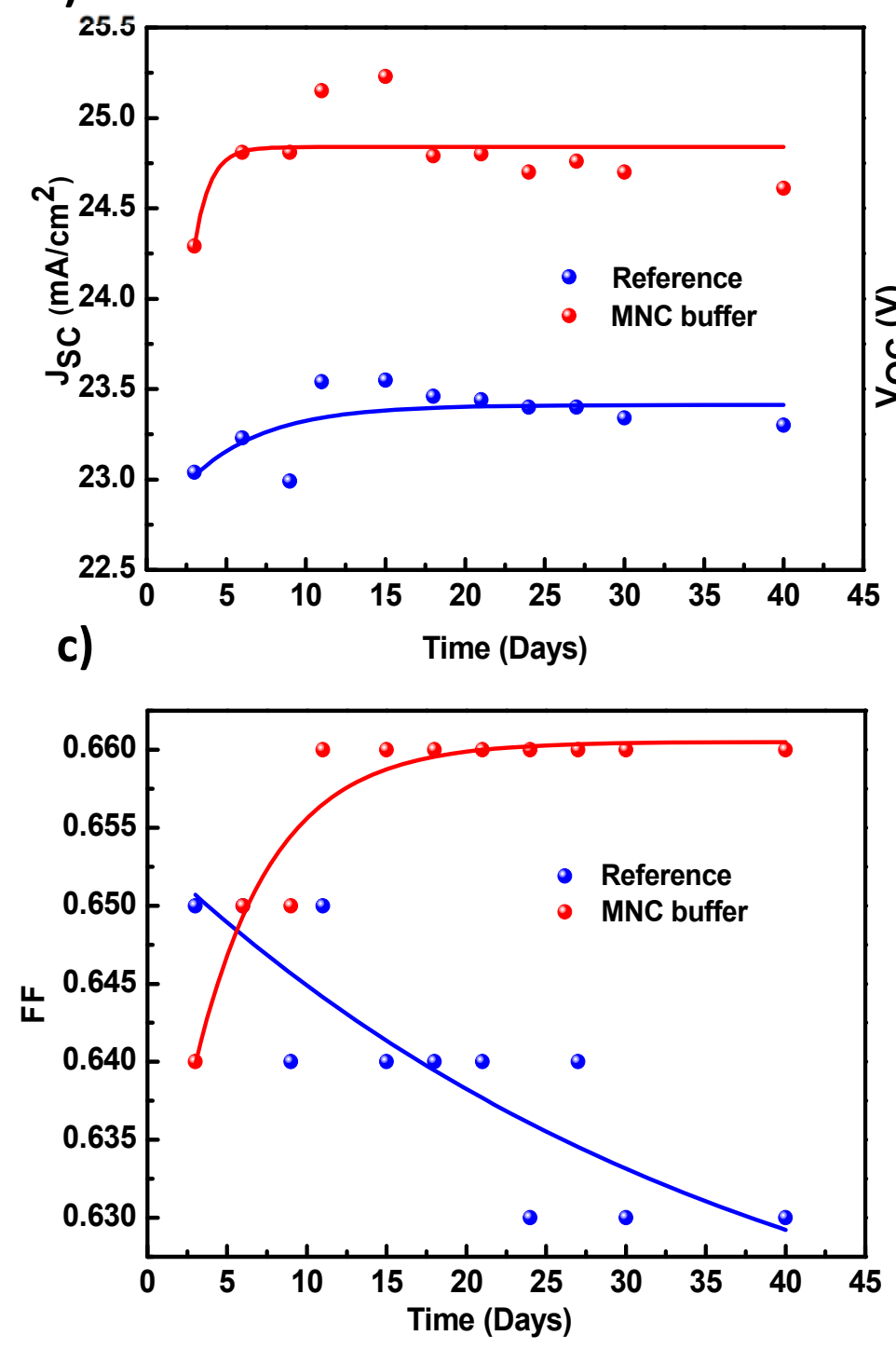

b)
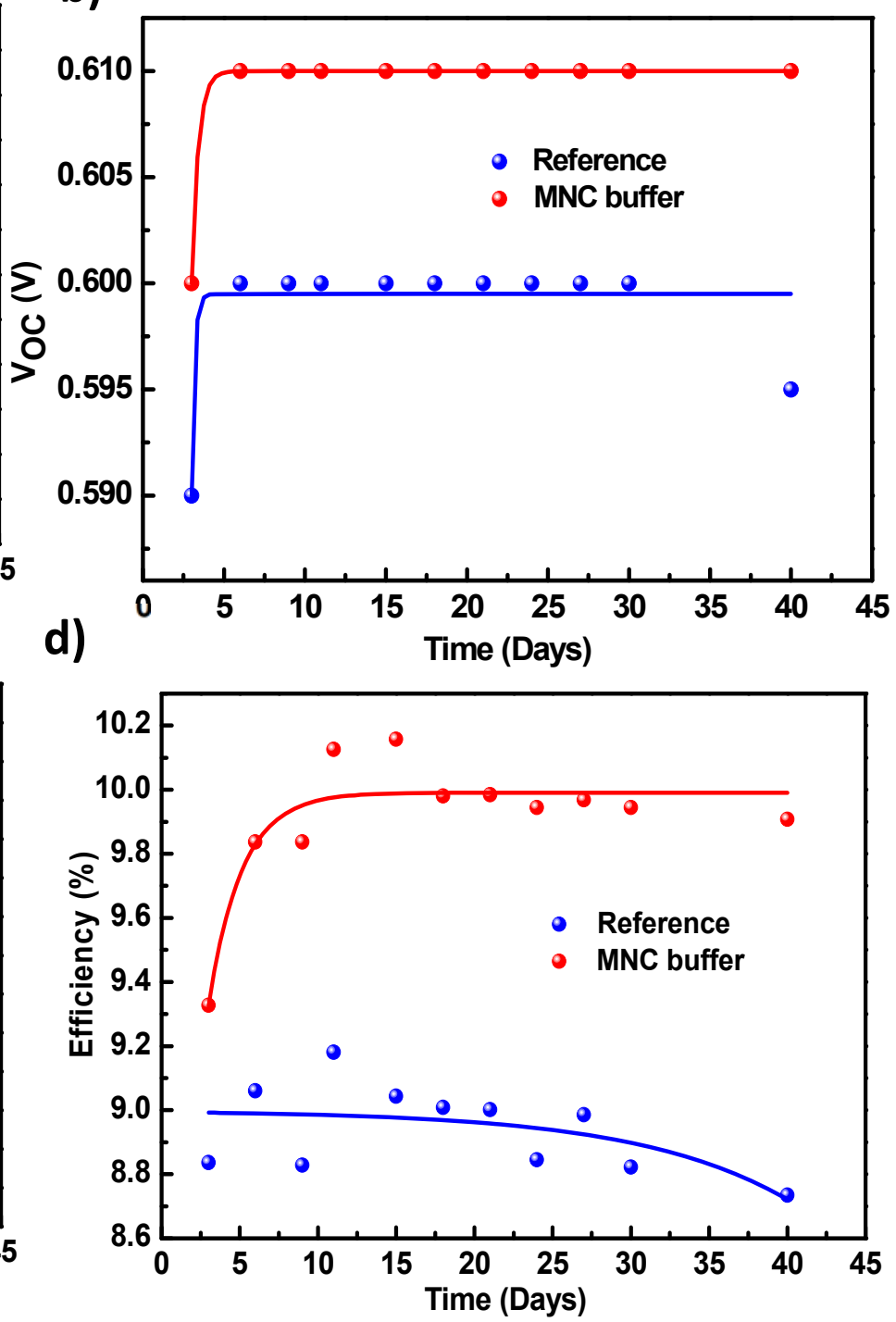

Figure 3: Comparison of the evolution of PV parameters ((a) JSC, (b) VoC, (c) FF, (d) Efficiency) between reference and MNC buffer layer inserted devices (champion devices in both cases) up to 40 days stored in ambient condition. 
a)
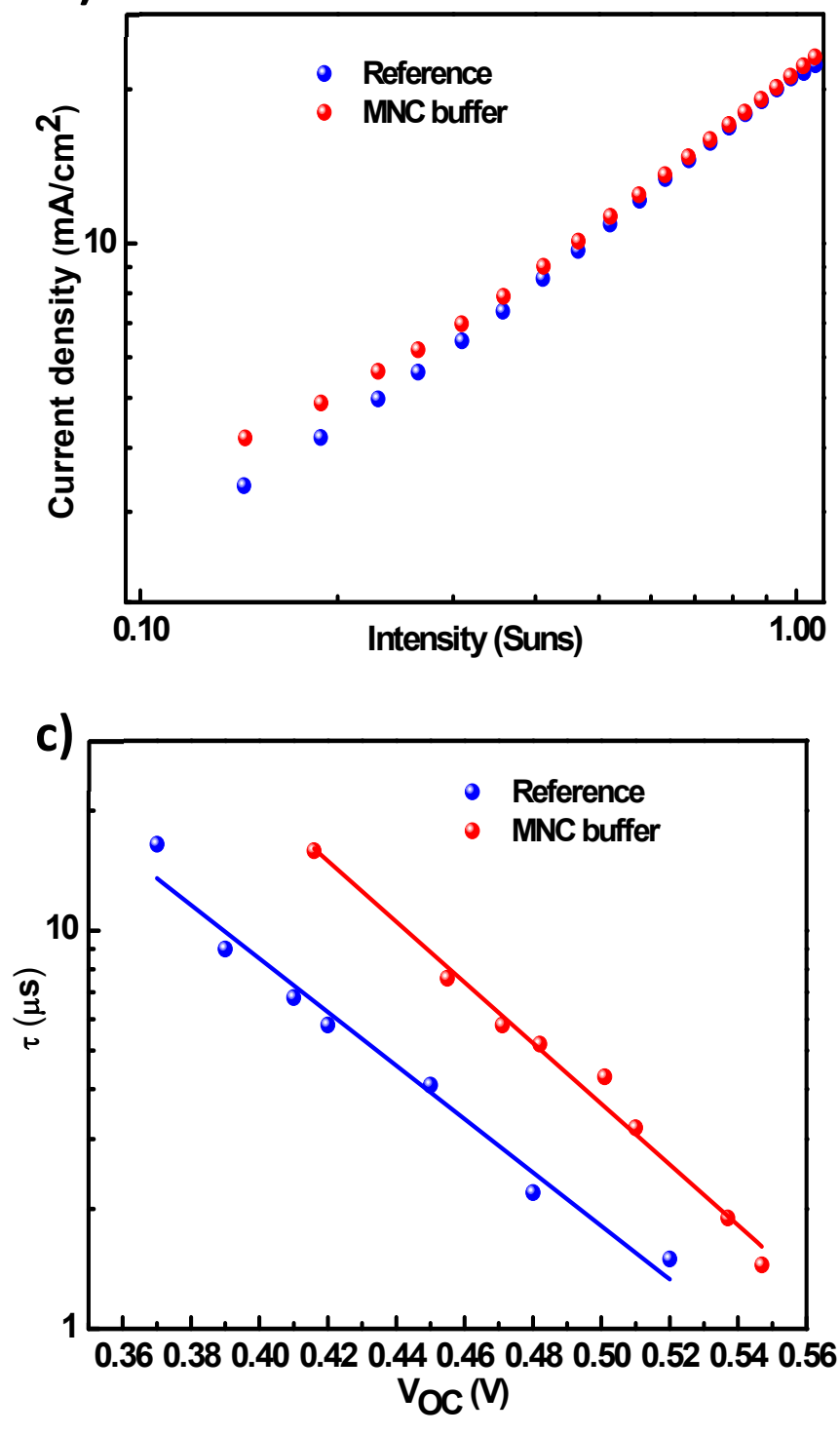

b)

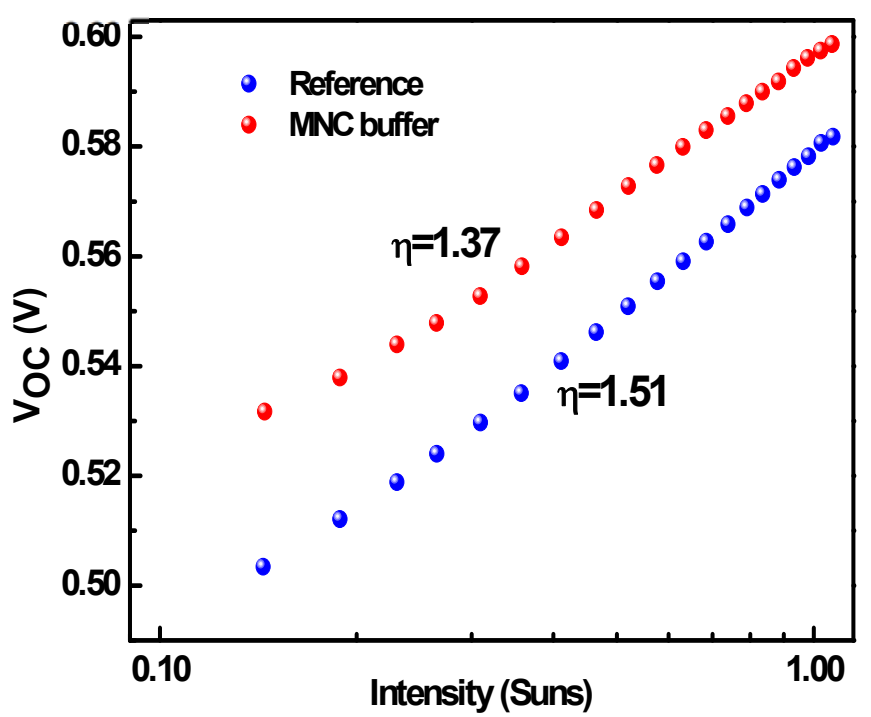

d)

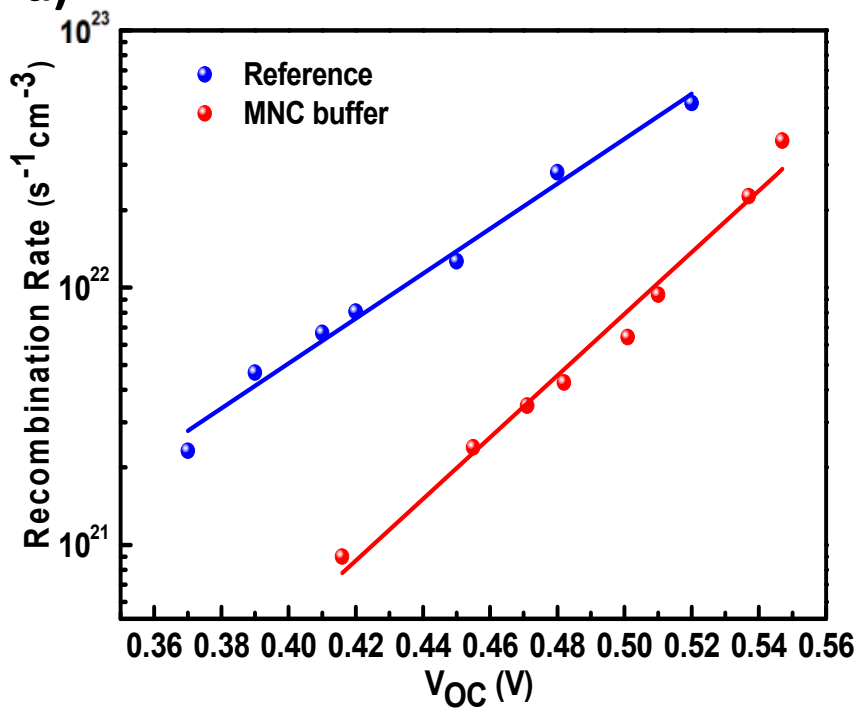

Figure 4: Intensity dependent (a) photocurrent and (b) Voc variation for Reference and MNC interface devices. (c) Comparison of recombination lifetime ( $\left.\boldsymbol{\tau}_{\mathbf{r}}\right)(\mathrm{d})$ Recombination rate (R) for both devices. 
TOC:

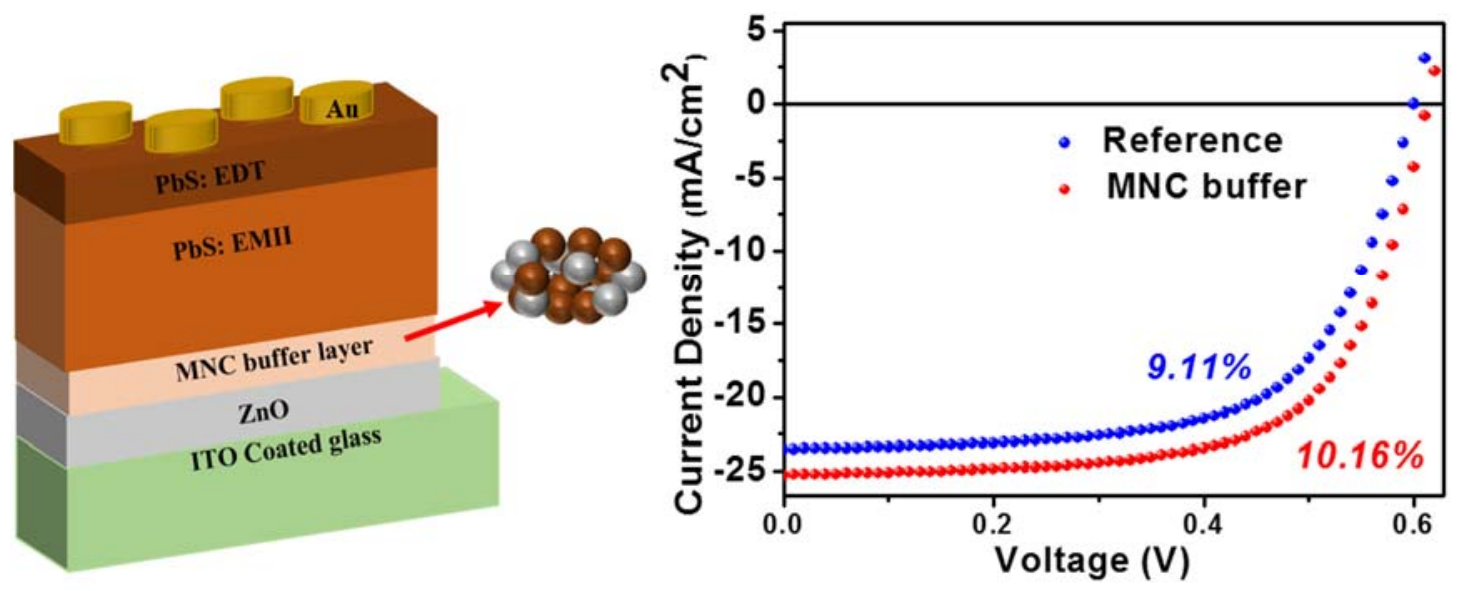

